\title{
RF/Analog and Linearity Performance Evaluation of Lattice-Matched ultra-thin AIGaN/GaN Gate Recessed MOSHEMT with Silicon Substrate
}

\section{Abdul Naim Khan ( $D$ 19pec001@Inmiit.ac.in )}

The LNM Institute of Information Technology https://orcid.org/0000-0002-2752-3122

KANJALOCHAN JENA

LNMIIT: The LNM Institute of Information Technology https://orcid.org/0000-0002-4705-962X

\section{Soumya Ranjan Routray}

SRMIST: SRM Institute of Science and Technology

Gaurav Chatterjee

LNMIIT: The LNM Institute of Information Technology

\section{Research Article}

Keywords: AIGaN/GaN, HEMT, MOSHEMT, GR-MOSHEMT, Leakage Current, lon/loff Current ratio

Posted Date: September 21st, 2021

DOI: https://doi.org/10.21203/rs.3.rs-913141/v1

License: (c) (i) This work is licensed under a Creative Commons Attribution 4.0 International License. Read Full License

Version of Record: A version of this preprint was published at Silicon on January 13th, 2022. See the published version at https://doi.org/10.1007/s12633-021-01605-3. 


\title{
RF/Analog and Linearity Performance Evaluation of Lattice- Matched ultra-thin AIGaN/GaN Gate Recessed MOSHEMT with Silicon Substrate
}

\author{
Abdul Naim Khan ${ }^{1}$, K. Jena ${ }^{1}$, S. Routray ${ }^{2}$, G. Chatterjee ${ }^{1}$ \\ ${ }^{1}$ Department of Electronics and Communication Engineering, The LNM Institute of Information Technology, \\ Jaipur, Rajasthan, 302031, India \\ ${ }^{2}$ Department of Electronics and Communication Engineering, SRM Institute of Science and Technology, \\ Chennai, Tamil Nadu, 603203, India
}

\begin{abstract}
-
In this article, the Authors have demonstrated and analyzed various analog/RF and linearity performance of a $\mathrm{AlGaN} / \mathrm{GaN}$ gate recessed MOSHEMT (GR-MOSHEMT) grown on a Si substrate with mathematical modeling based TCAD simulation. Specifically, a $\mathrm{Al}_{2} \mathrm{O}_{3}$ dielectric GR-MOSHEMT has shown tremendous potential in terms of AC/DC figure of merits (FOM's) such as low leakage current, high transconductance, high $\mathrm{I}_{\mathrm{on}} / \mathrm{I}_{\mathrm{off}}$ current ratio and excellent linear properties corresponding to conventional AlGaN/GaN HEMT and MOSHEMT. The figure-of-merit metrics such as VIP2, VIP3, IIP3 and IDM3 are performed for different drain to source voltages $\left(\mathrm{V}_{\mathrm{DS}}\right)$ of $2.5 \mathrm{~V}, 5 \mathrm{~V}$ and $10 \mathrm{~V}$. All the modeling and simulation results are generated by Commercial Silvaco TCAD and found to be satisfactory in terms of high frequency and power applications. The present GR-MOSHEMT device shows a superior performance with a threshold voltage of $0.5 \mathrm{~V}$, Current density of $888 \mathrm{~mA}$, high transconductance of $225 \mathrm{mS} / \mathrm{mm}$ and high unit gain cut-off frequency of $0.91 \mathrm{GHz}$. The results of the developed $\mathrm{AlGaN} / \mathrm{GaN}$ GR-MOSHEMT considerably improves the device performance and also suitable for high power distortion less RF applications.
\end{abstract}

Index terms - AIGaN/GaN, HEMT, MOSHEMT, GR-MOSHEMT, Leakage Current, Ion/I $I_{\text {off }}$ Current ratio

\section{INTRODUCTION}

Recently, AlGaN/GaN HEMT based devices have achieved much recognition for high power and high-frequency applications due to high breakdown voltage [1], high thermal conductivity, high saturation velocity, low effective mass and high two-dimensional electron gas (2DEG) at the GaN/AlGaN interface [2-7]. But the performance of HEMT based devices is limited in RF power applications due to high gate leakage and drain leakage current. AlGaN/GaN-based heterostructures provide 2DEG (2-dimensional electron Gas) at AlGaN-barrier and GaNChannel layer interface, which provides higher carrier mobility and current density generation of Spontaneous and Piezoelectric Polarizations. Recently, AlGaN/GaN based metal-oxidesemiconductor MOSHEMT with an insulating dielectric oxide layer $\left(\mathrm{Al}_{2} \mathrm{O}_{3}\right)$ primarily targeted the RF power applications due to its low gate leakage current and improved drain and threshold voltage [8-12]. But HEMT and MOSHEMT based devices suffer from high contact and high on-resistance due to metallic ohmic contacts and significant source/drain distance. However, the modified gate structure reduces the gate resistance while maintaining the modified gate 
length and reduced the gate capacitance [13-17]. Generally, AlGaN/GaN HEMTs are normally-on devices and operates in depletion mode. The corresponding MOSHEMTs with an extra dielectric between metal gate and thin barrier also operate as on-device but the threshold voltage moves towards positive compared to HEMT based on-devices [18, 19]. However, Gate recessed MOSHEMTs generally work as normally off device and operates in enhancement mode. These normally-off devices are more suitable in case of high power amplifier circuits, high power switching applications, and millimeter-wave applications due to their high gate dielectric, low leakage current and positive threshold voltage [20, 21].

There are many literature works available about modeling and simulation of analog and linearity performance of AlGaN/GaN HEMT [22-24]. However, as per the Author's knowledge, in depth analytical modeling and simulation work is yet to be done for conventional MOSHEMT and gate recessed MOSHEMT as evident in the literature explaining the RF and linearity parameters.

In this work, we have demonstrated the lattice-matched AlGaN/GaN based HEMT, MOSHEMT and gate recessed MOSHEMT to analyze and compare the analog and linearity performances. Section II describes the device structures and simulation models. In Section III, the simulated results are compared with experimental results along with physical models. Finally, the conclusion is drawn in Section IV.

\section{Device Structure and Modeling Equations}

\subsection{Device Structure}

Fig. 1 shows the cross-sectional schematics of the conventional AlGaN/GaN HEMT, normally on MOSHEMT and normally off gate recessed MOSHEMT. The GaN-Channel, AlGaN-barrier and AlN-nucleation layer thicknesses in all the devices are $1.47 \mu \mathrm{m}, 20 \mathrm{~nm}$ and $1 \mathrm{~nm}$ respectively. The $\mathrm{Al}$ composition in the $\mathrm{AlGaN}$ layer is kept at 0.3 to maintain good interface property between $\mathrm{AlGaN}$ and $\mathrm{GaN}$ [25-27]. In case of MOSHEMT, $\mathrm{Al}_{2} \mathrm{O}_{3}$ is used as a gate dielectric with a thickness of $5 \mathrm{~nm}$ to minimize the leakage current density. The thin $\mathrm{Al}_{2} \mathrm{O}_{3}$ dielectric oxide layer is used due to its relatively high band gap $(9 \mathrm{eV})$, high dielectric constant $(\mathrm{k} 10)$, high breakdown field $(107 \mathrm{~V} / \mathrm{cm})$ and good thermal stability. The dielectric oxide reduces the gate leakage current when it contacts under the gate contact, which allows the application of high positive gate voltage further to increase the sheet electron density in 2DEG channel. AlGaN works as a barrier layer with $0.3 \mathrm{Al}$ composition and $\mathrm{GaN}$ as a channel layer in the device. The purpose of inserting nucleation layer between the GaN Channel and substrate to 
minimize the stress and related lattice mismatch. There is a strong confinement of 2DEG at the $\mathrm{AlGaN} / \mathrm{GaN}$ interface due to spontaneous and piezoelectric polarization charges [26]. Donors are created near the source and drain junctions to reduce the access and contact resistance. The Gate and metal-electrodes (for Source/Drain) work functions are $5 \mathrm{eV}$ and $3.9 \mathrm{eV}$ (Hafnium) respectively. The gate-source spacing $\left(\mathrm{L}_{\mathrm{GS}}=2.5 \mathrm{um}\right)$ and gate-drain spacing $\left(\mathrm{L}_{\mathrm{GD}}=2.5 \mathrm{um}\right)$ are kept equal in all the device structures. Silicon substrate is used to achieve excellent thermal characteristics.

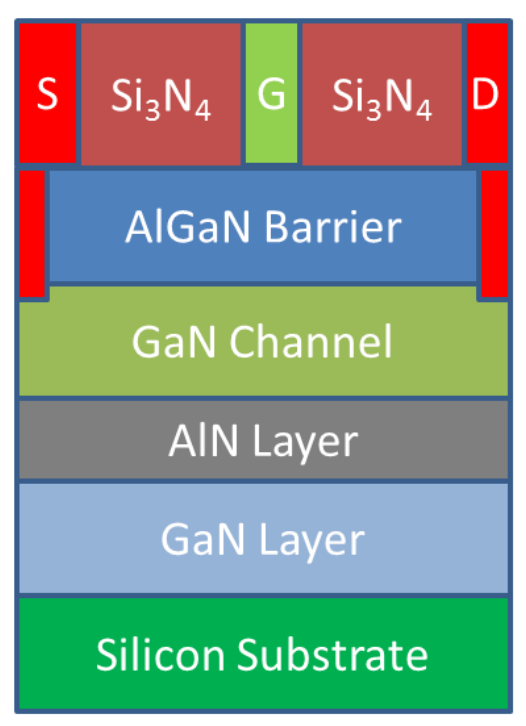

a

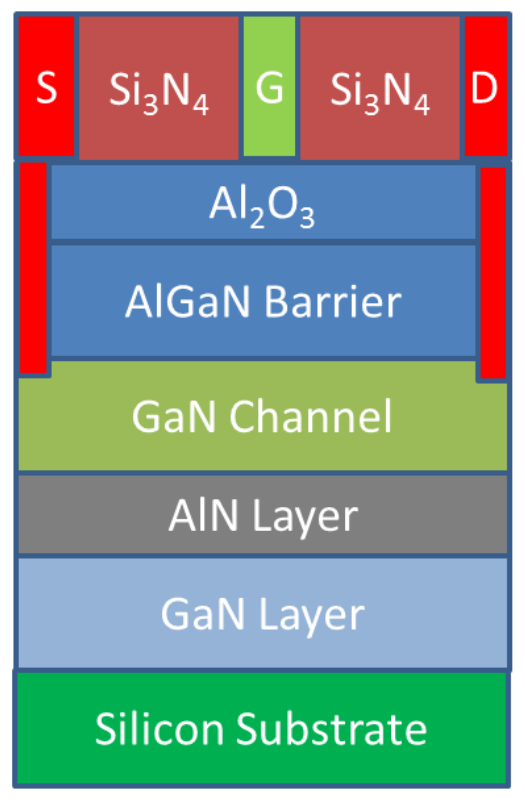

b

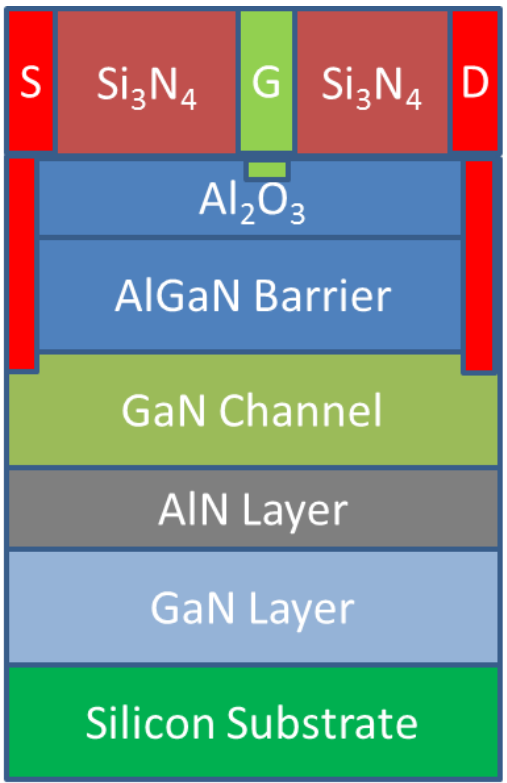

C

Fig.: 1 Device structures of (a) Conventional AlGaN/GaN HEMT (b) Normally on AlGaN/GaN MOSHEMT (c) Normally off Gate recessed AlGaN/GaN MOSHEMT

\subsection{Modeling Equations}

\section{A. Calculation of sheet charge density due to Polarization}

Spontaneous and piezoelectric polarization charges exist at the boundary of the $\mathrm{Al}_{2} \mathrm{O}_{3} / \mathrm{AlGaN}$ and $\mathrm{AlGaN} / \mathrm{GaN}$ interface. To calculate the spontaneous and piezoelectric polarization charges at the heterogeneous interface, the following equations (1) - (9) have been used [27].

$$
\begin{aligned}
& |\sigma(x)|=\left|P_{P E}\left(A l_{x} G a_{1-x} N\right)+P_{S P}\left(A l_{x} G a_{1-x} N\right)-P_{S P}(G a N)\right| \\
& |\sigma(x)|=\left|2 \frac{a(0)-a(x)}{a(x)}\left\{e_{13}(x)+e_{33}(x) \frac{C_{13}(x)}{C_{33}(x)}\right\}+P_{S P}(x)-P_{S P}(0)\right| \\
& a(x)=(-0.077 x+3.189) 10^{-10}
\end{aligned}
$$


Where $\sigma(x)$ and $a(x)$ is sheet charge density and lattice constant respectively.

$a(0)=a_{G a N}$

and $C_{13}, C_{33}$ are the elastic constant, $e_{13}$ and $e_{33}$ are the piezoelectric constants given as follows:

$C_{13}(x)=(5 x+103)$

$C_{33}(x)=(-32 x+405)$

$e_{13}(x)=(-0.11 x-0.49)$

$e_{33}(x)=(0.73 x+0.73)$

The spontaneous polarization of $A l_{x} G a_{1-x} N$ is also a function of the Al mole fraction $x$ and is given by:

$$
P_{S P}(x)=(-0.052 x-0.029)
$$

The extensive simulations are done using Commercial Silvaco TCAD [28] with Newton numerical methods and the models such as Gansat, SRH (Shockley-Read-Hall recombination), Albrct, and spontaneous and piezoelectric polarization are taken in to consideration. The drift-diffusion model has been taken in to consideration for the carrier transport in the channel of devices. A variation of carrier mobility with doping concentration is established by low-field mobility model. The mesh value of the device structures for the crucial regions is chosen precisely for accurate simulation and accelerating computational efficiency.

\section{B. Calculations of Electrical and thermal parameters for $A l_{x} G a_{1-x} N$ :}

The energy bandgap of III-Nitride binary compounds are calculated as a function of temperature using [29]:

$$
\begin{gathered}
E_{g}(G a N)=3.507-\frac{0.909 \times 10^{-3} T^{2}}{T+830} \\
E_{g}(A l N)=6.23-\frac{1.799 \times 10^{-3} T^{2}}{T+1462}
\end{gathered}
$$

Now, Vegard's law is used for finding the bandgap energy of the ternary compound [3031]. 


$$
E_{g}\left(A l_{x} G a_{1-x} N\right)=x E_{g}(A l N)+(1-x) E_{g}(G a N)-b x(1-x)
$$

Where $\mathrm{b}$ and $\mathrm{x}$ is the bowing parameter and $\mathrm{Al}$ mole fraction respectively

The electron affinity is calculated by properly maintain the band edge offset ratio and is given by [32].

$$
\frac{\Delta E_{c}}{\Delta E_{v}}=\frac{0.7}{0.3}
$$

Electron affinity and permittivity of $A l_{x} G a_{1-x} N$ is computed as by the following equations [33]:

$$
\begin{aligned}
& \chi\left(A l_{x} G a_{1-x} N\right)=\chi(G a N)-1.89 x+0.91 x(1-x) \\
& \varepsilon\left(A l_{x} G a_{1-x} N\right)=8.5 x+8.9(1-x)
\end{aligned}
$$

The ternary compound materials density of states masses a function of mole fraction associated with compound material and is given by linear interpolations [29]:

$$
\begin{aligned}
& m_{e}\left(A l_{x} G a_{1-x} N\right)=0.314 x+0.2(1-x) \\
& m_{h}\left(A l_{x} G a_{1-x} N\right)=0.417 x+1.0(1-x)
\end{aligned}
$$

Where $m_{e}$ and $m_{h}$ are the electron and hole mass density of $A l_{x} G a_{1-x} N$ material.

\section{Calculation of Analog/RF Parameters for HEMT and MOSHEMT}

Transconductance should be high for High linearity devices to achieve maximum gain. Generally, it is divided into the gate and drain transconductances. Most of the device provides the flat transconductance. Still, in HEMT and MOSHEMT structures, it shows the bell-shaped curve due to the self-heating thermal effects, low to moderate drain voltage and nonlinearity source/drain resistances. Gate transconductance $\left(\mathrm{g}_{\mathrm{m}}\right)$ is the ratio of the change in ID concerning the change in VGS with constant VDS. In the same way, output or drain conductance $\left(\mathrm{g}_{\mathrm{ds}}\right)$ is the ratio of change in drain current to the drain voltage and constant gate voltage. Mathematical equations used for the measurement of transconductances are [34]:

$$
\begin{aligned}
& g_{m}=\frac{\partial I_{D}}{\partial V_{G S}} \mid V_{D S}=\mathrm{const} \\
& g_{d s}=\frac{\partial I_{D}}{\partial V_{D S}} \mid V_{G S}=\mathrm{const}
\end{aligned}
$$


Primarily there are two types of capacitance effects which are Gate-to-source and gate-todrain capacitance. For gate-to-source capacitance, the variation of gate charge for change in the gate voltage and gate charge variation with change in drain voltage is referred to as gate-to-drain capacitance. Gate-to-source and gate-to-drain capacitances are represented as [34]:

$$
\begin{aligned}
& C_{g s}=\frac{\partial Q}{\partial V_{g s}} \\
& C_{g d}=\frac{\partial Q}{\partial V_{d s}}
\end{aligned}
$$

The cut-off frequency is proportional to the transconductance. It is also inversely proportional to the summation of the intrinsic capacitances of gate-to-drain and gate to source. The maximum oscillation frequency varies by the cut-off frequency. It also depends on various factors like gate-on resistance and gate-to-drain capacitance. In case of MOSHEMT the reduced on-resistance and gate capacitance leads to increase in the Oscillation frequency. Mathematically equations related to the cut off and maximum oscillation frequencies are expressed as $[35,36]$ :

$$
f_{T}=\frac{g_{m}}{\left(2 \times \pi \times C_{g g}\right)}
$$

Where

$$
\begin{aligned}
& C_{g g}=C_{g d}+C_{g s} \\
& f_{\max }=\frac{f_{T}}{\left(2 \times \sqrt{g_{d s} \times\left(R_{g}+R_{s}\right)+2 \pi \times f_{T} \times R_{g} \times C_{g d}}\right)}
\end{aligned}
$$

Where

$$
R_{g}=\frac{1}{G_{g g}}
$$

\section{Intermodulation distortion and linearity performance parameters}

In Analog/RF-based circuits, Intermodulation distortion and higher-order harmonics are introduced at the output end, which causes the degradation of device performance by reducing the output power component. For better device performance, distortion and 
harmonics types of error must be removed. Linearity Characteristics can be analyzed using different types of parameters like High order trans-conductance, interception points, and intermodulation distortion for smooth device operation.

For a Linear device, Transconductance and its higher-order coefficients should be expressed by following modeling equation (26) -(30) [37]:

$$
g_{m n}=\frac{1}{n !} \frac{\partial^{n} I_{d}}{\partial V_{g s}^{n}}, \text { where } n=1,2,3
$$

Different Figure of merits (FOM's) is used in this paper to analyze the AlGaN/GaN based devices' behavior in linearity mode $[38,39]$. Second-order intercept point ( $\left.\mathrm{V}_{\mathrm{IP} 2}\right)$ and Third-order intercept point $\left(\mathrm{V}_{\mathrm{IP} 3}\right)$ are defined as the extrapolated input voltage and gate voltage respectively at which the second-order and third-order harmonics become equal to the fundamental tone in the device's drain current (Id). So II-order and IIIorder harmonics, $\mathrm{V}_{\mathrm{IP} 2}$ and $\mathrm{V}_{\mathrm{IP} 3}$, are mathematically represented as follows:

$$
\begin{aligned}
& V I P 2=4 \times \frac{g_{m 1}}{g_{m 2}} \\
& V I P 3=\sqrt{24 \times \frac{g_{m 1}}{g_{m 3}}}
\end{aligned}
$$

The interpolated input power at which I-order and III-order harmonics power are equal is given by the Input intercept point of the third harmonic (IIP3). Intermodulation distortion of third harmonic is defined for nonlinear devices when two or more signals are mixed. Mathematically IIP3 and IDM3 are represented as:

$$
\begin{aligned}
& I I P 3=\frac{2}{3} \times \frac{g_{m 1}}{g_{m 3} \times R_{s}} \\
& I D M 3=\left[\frac{9}{2} \times(V I P 3)^{2} \times g_{m 3}\right]^{2} \times R_{s}
\end{aligned}
$$

All the above mention FOM's are used to differentiate between the proposed $\mathrm{AlGaN} / \mathrm{GaN}$ gate recessed MOSHEMT device structure for different changes in drainto-source voltages in terms of linearity performance and intermodulation distortions. 


\section{Simulation Results and Discussions}

The accuracy and efficiency of a device depends on proper meshing, dimensions and precise selection of the parameters with minute observations. So, a fine meshing at the important region of operations such as channel and source/drain edge is chosen for the device simulations. Results are generated for all the proposed structures and verified with the data available in literature.

\subsection{DC analysis of the AIGaN/GaN HEMT, MOSHEMT and GR-MOSHEMT}
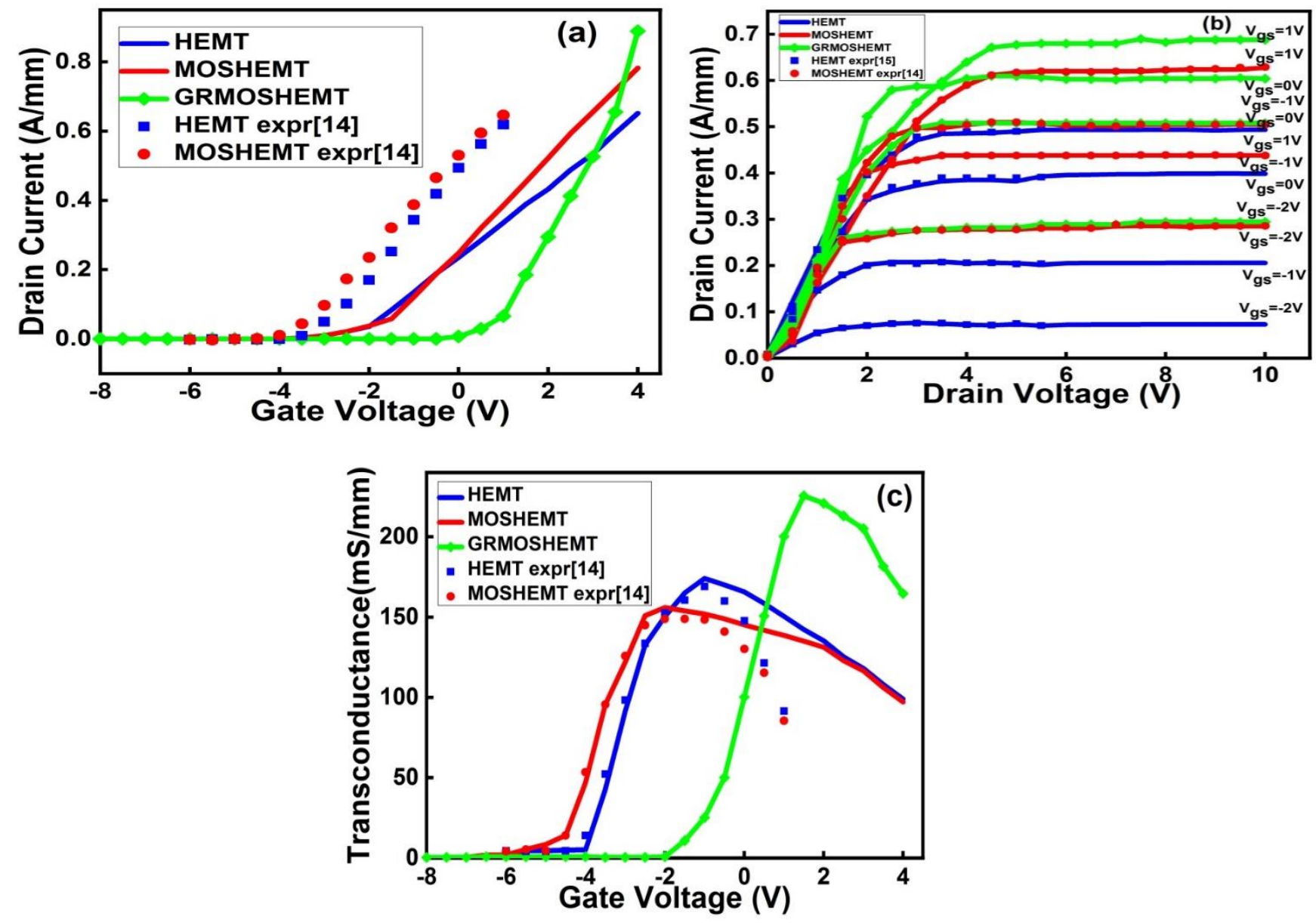

Fig.2 Comparative DC analysis curves between HEMT, MOSHEMT and gaterecessed MOSHEMT (a) $I_{d}-V_{g}$ characteristic curves (b) $I_{d}-V_{d}$ characteristic curves (c) $\mathrm{g}_{\mathrm{m}}-\mathrm{V}_{\mathrm{g}}$ characteristic curves

Fig. 2 represents the Transfer, Output and Transconductance characteristics of the Conventional HEMT, MOSHEMT and Gate Recessed MOSHEMT. Fig. 2(a) represents the transfer characteristics of AlGaN/GaN HEMT, MOSHEMT and Gate recessed MOSHEMT with fixed oxide and barrier layer thickness. The recessed gate shows a higher drain current than HEMT and MOSHEMT due to adding an extra dielectric oxide layer with the change in gate structure. It is observed that Recessed gate MOSHEMT achieved a maximum 
current density of $888 \mathrm{~mA} / \mathrm{mm}$ compared to $651 \mathrm{~mA} / \mathrm{mm}$ (in case of HEMT) and 781 $\mathrm{mA} / \mathrm{mm}$ (in case of MOSHEMT) due to the structural modification of gate. If Ion current increases sustainable way, it shows better improvement in the simulation results and device performance. The threshold voltage obtained for HEMT, MOSHEMT and gate recessed MOSHEMT are $-2.18 \mathrm{~V},-1.23 \mathrm{~V}$ and 0.5 respectively from the Ids vs. Vgs curve in linear scale. Fig. 2(b) shows the output characteristics curve of differently structured devices. The maximum current density of recessed gate MOSHEMT is $687 \mathrm{~mA} / \mathrm{mm}$ for $\mathrm{Vgs}=1 \mathrm{~V}$, which is $28.2 \%$ and $8.5 \%$ higher compared to HEMT and MOSHEMT respectively. From this curve, we observed that a reduction in gate length and increment in width of gate recessed MOSHEMT structure leads to an increase in DC characteristics. Fig. 2(c) shows the transconductance $\left(\mathrm{g}_{\mathrm{m}}\right)$ of differently structured devices. It is calculated from the derivative of drain current to gate voltage at fixed drain voltage and expressed in Siemens. The high peak extrinsic $\mathrm{g}_{\mathrm{m}}$ of gate recessed MOSHEMT was $255 \mathrm{mS} / \mathrm{mm}$ which is much higher than HEMT and MOSHEMT devices. Gate controlling ability of recessed gate MOSHEMT increases due to reduction in the distance between gate and GaN-channel, so it shows better improvement than HEMT and MOSHEMT. So gate recessed MOSHEMT peak transconductance increases arbitrarily as compare to HEMT and MOSHEMT device structures. Threshold voltage and transconductance of recessed gate MOSHEMT show a strong impact due to the dielectric layer of $\mathrm{Al}_{2} \mathrm{O}_{3}(5 \mathrm{~nm})$ inserted between the gate and the $\mathrm{AlGaN}$ barrier layer and changes in gate structure.

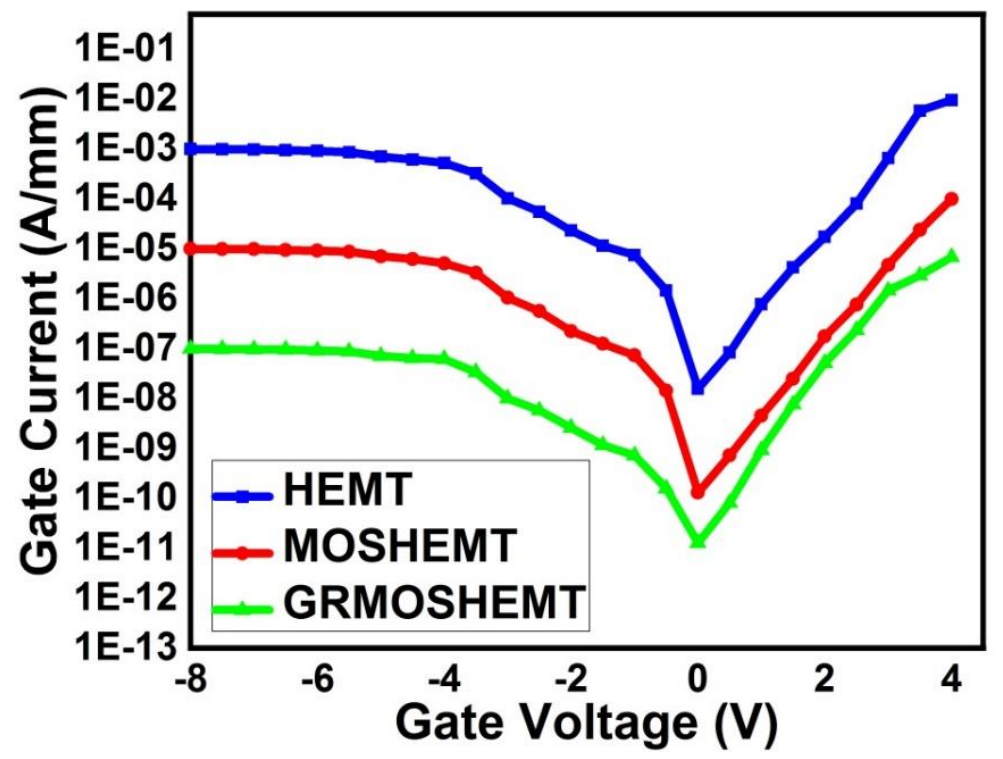

Fig.3 Comparative analysis of Gate Leakage current between AlGaN/GaN HEMT, MOSHEMT and Gate recessed MOSHEMT 
Fig. 3 shows a comparison of the gate leakage current performance of GaN/AlGaN HEMT, MOSHEMT and gate recessed MOSHEMT with the exact device dimensions in the $\log$ scale. The leakage current of gate recessed MOSHEMT is found to be significantly lower than that of HEMT and MOSHEMT. The density of gate leakage current of gate recessed MOSHEMT is almost 2-3 orders of magnitude lower than that of the MOSHEMT. Similarly, this way, the HEMT gate leakage current is almost 2-3 orders of magnitude lower than MOSHEMT. It is observed that, there is an improvement in drain current due to excellent insulating properties of $\mathrm{Al}_{2} \mathrm{O}_{3}$ for MOSHEMT and gate recessed MOSHEMT.

The insertion of $\mathrm{Al}_{2} \mathrm{O}_{3}$ between the gate electrode and $\mathrm{AlGaN}$ barrier layer will work as an efficient gate insulator and decreased gate leakage current compared to HEMT. Furthermore, modification of gate electrodes also reduced the gate leakage current for gate recessed MOSHEMT. It is well known that Off-state drain-leakage current is an essential factor for the device's performance. Drain leakage current depends on the trap energy and density level of the device. So we can say that the drain leakage current is minimum in MOSHEMT devices compared to the HEMT based devices.

\subsection{AlGaN/GaN gate recessed MOSHEMT Analog/RF performance}

Fig.4 shows the analog/RF behavior of AlGaN/GaN gate recessed MOSHEMT. Fig.4 (a) shows the variations of drain current with respect to gate-to-source voltage of GaN/AlGaN gate recessed MOSHEMT for different drain-to-source voltages. It shows that higher current density $888 \mathrm{~mA} / \mathrm{mm}$ is achieved when $\mathrm{V}_{\mathrm{ds}}=10 \mathrm{~V}$ as compare to $\mathrm{V}_{\mathrm{ds}}=5 \mathrm{~V}$ and $2.5 \mathrm{~V}$. It is found that there is High transconductances of gate recessed MOSHEMT for $\mathrm{V}_{\mathrm{ds}}=10 \mathrm{~V}$ as compared to $\mathrm{V}_{\mathrm{ds}}=2.5 \mathrm{~V}$ and $5 \mathrm{~V}$ due to sustainable increases in $\mathrm{ON}$ Current is as shown in Fig.4 (b). In strong inversion region transconductance linearly increases with increment of drain-to-source voltages. Fig.4 (c) shows the intrinsic parasitic gate-to-source capacitance $\left(\mathrm{C}_{\mathrm{gs}}\right)$ which is plotted with gate-to-source voltage $\left(\mathrm{V}_{\mathrm{gs}}\right)$ for different drain-to-source voltages. Parasitic capacitances depend on bias conditions so there is variation in the values

with changes in drain voltages. As seen in figure the parasitic capacitance shows a decrement with respect to increasing in drain-to-source voltages because channel is tapered and pinched off near the drain region so channel will not be uniform and it shows a decrement when drain voltages increases. 

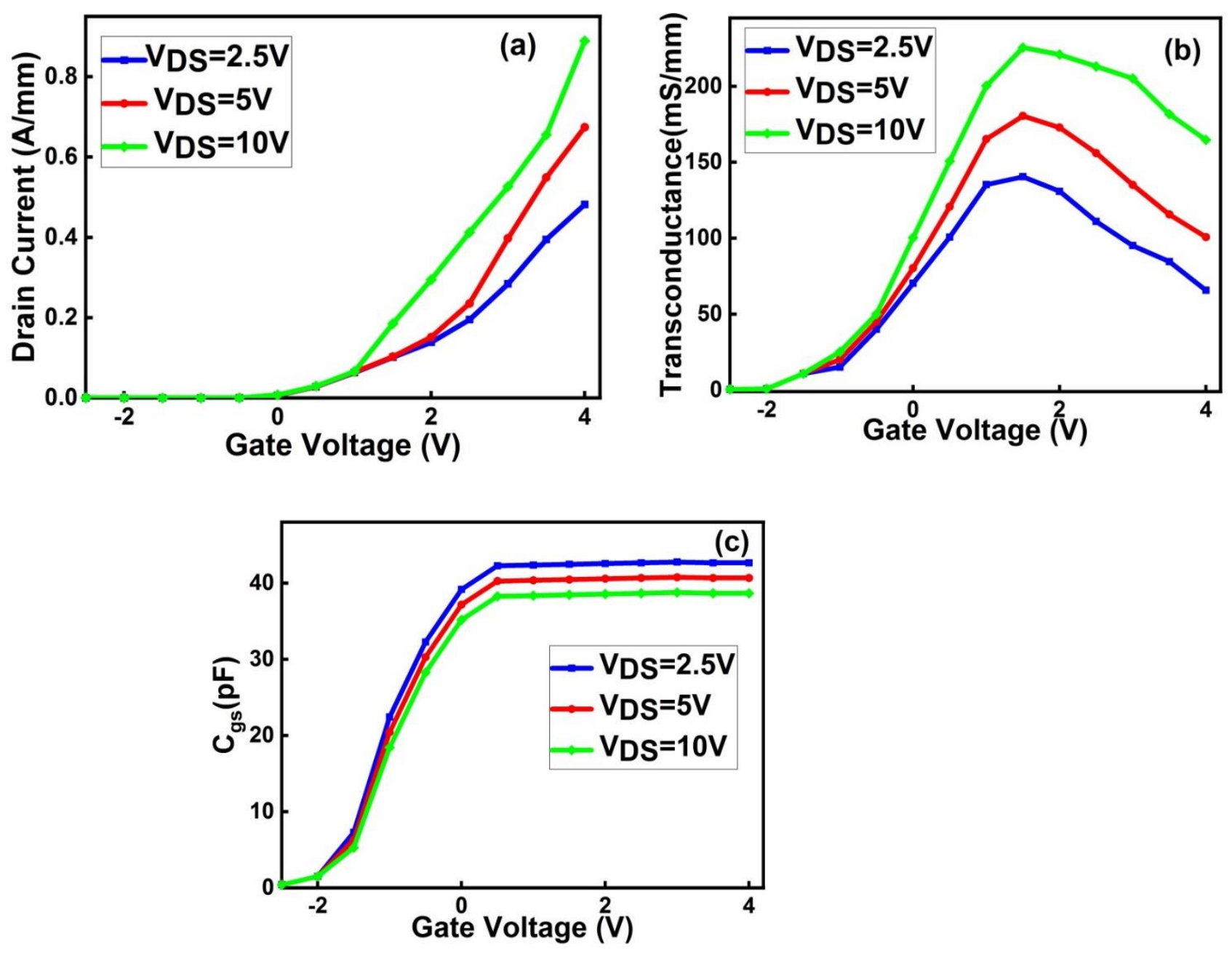

Fig.4 Performance parameters analysis of gate recessed MOSHEMT for different drain voltages (a) $I_{d}-V_{g}$ characteristic curves (b) $g_{m}-V_{g}$ characteristic curves (c) $C_{g s}-V_{g}$ characteristic curves

Transconductance and gate-to-source capacitance behave oppositely with respect to increment in gate-to-source voltage. The unit gain cut-off frequencies $\left(\mathrm{f}_{\mathrm{T}}\right)$ are plotted with respect to gate-to-source voltage $\left(\mathrm{V}_{\mathrm{gs}}\right)$ for different drain-to-source voltages in Fig.5 (a). It shows that cut-off frequency shows much better improvement when drain-to-source voltage increases linearly. Fig.5 (b) shows the variation of Maximum Oscillation frequency with respect to the gate-to-source voltage for different drain-to-source voltages. Maximum oscillation frequency is proportional to the cut-off frequency and anti-proportional to the gate-to-drain capacitance so it shows relative upward movements with respect to increasing in drain-to-source voltages. 

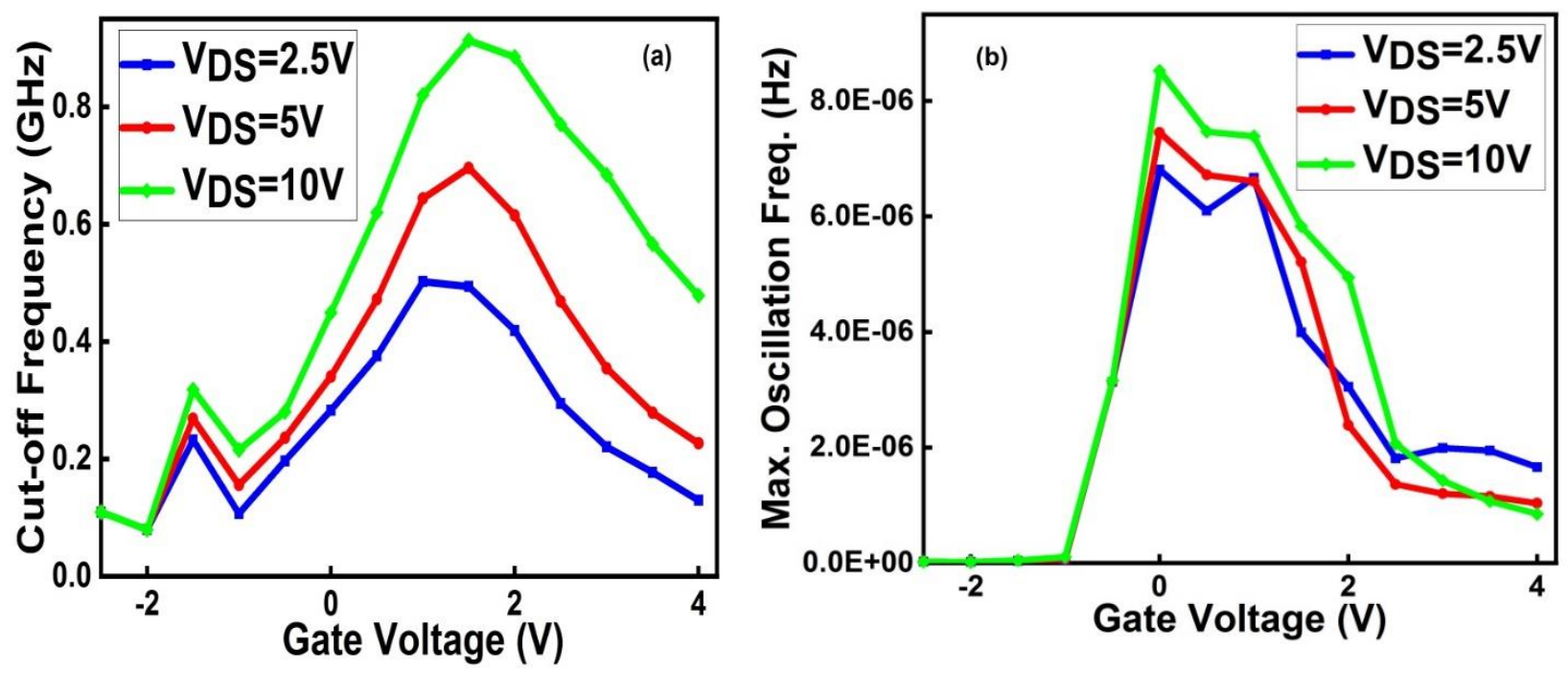

Fig.5 RF Performance parameters analysis of gate recessed MOSHEMT for different drain voltages (a) $f_{T}-V_{g}$ characteristic curves (b) $F_{M a x}-V_{g}$ characteristic curves

\subsection{Improved linearity Parameters of the AIGaN/GaN GR-MOSHEMT:}

Device Linearity is the basic requirement for proper functioning of electronic circuit elements. Different FOM's parameters for linearity are $\mathrm{g}_{\mathrm{m} 2}, \mathrm{~g}_{\mathrm{m} 3}, \mathrm{~V}_{\mathrm{IP} 2}, \mathrm{~V}_{\mathrm{IP} 3}$, IIP3 and IDM3. High FOMs determines the better device performance. Fig.6, 7 and 8 shows the different FOM's parameters curves for proposed models.

Fig.6 shows the variation of second order and third order differential of transconductances, $\mathrm{g}_{\mathrm{m} 2}$ and $\mathrm{g}_{\mathrm{m} 3}$ with respect to gate-to-source voltage for different drain-to-source voltages.
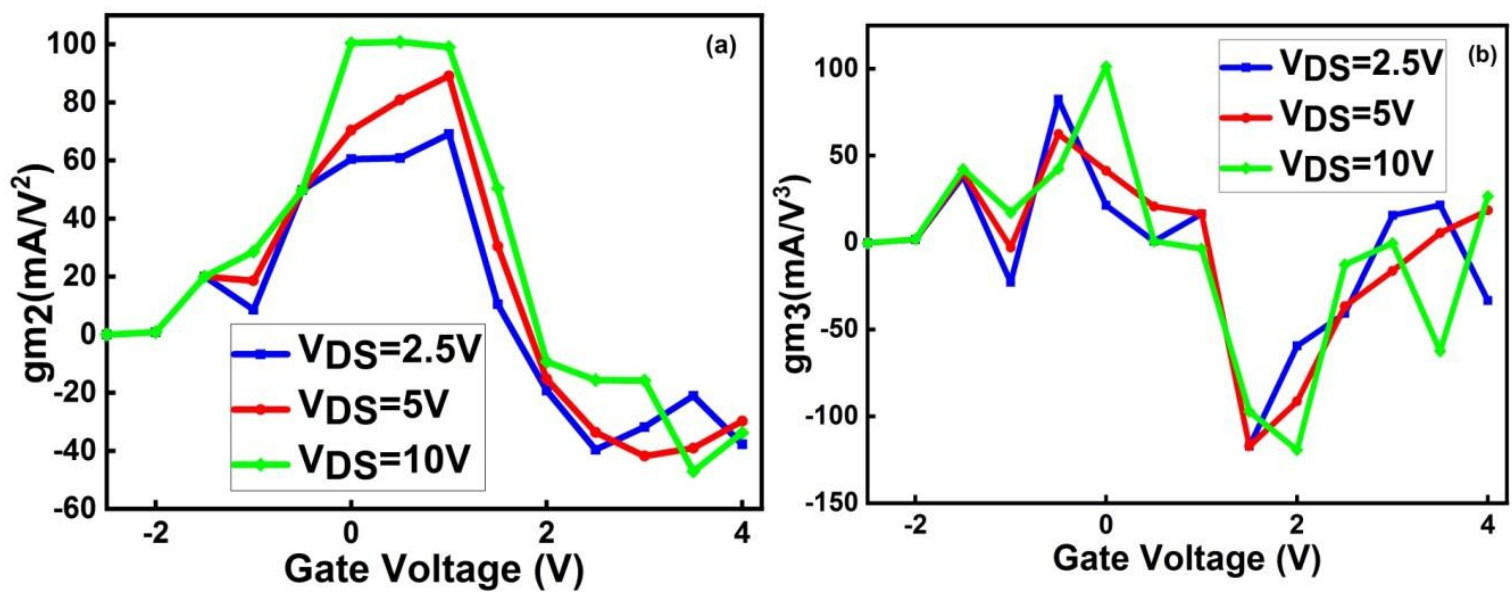

Fig.6 Trans conductance parameter analysis of gate recessed MOSHEMT for different drain voltages (a) $g_{m 2}-V_{g}$ characteristic curves (b) $g_{m 3}-V_{g}$ characteristic curves

For low distortion and higher linearity a high value of $\mathrm{V}_{\text {IP2 }}$ and $\mathrm{V}_{\text {IP3 }}$ is required. When gate is in moderate region than it will generate a peak for low gate bias. Second and third order 
Voltage intercepts points $\mathrm{V}_{\text {IP2 }}$ and $\mathrm{V}_{\text {IP3 }}$ with respect to gate-to-source voltage for variation in drain-to-source voltages is shown in Fig.7.
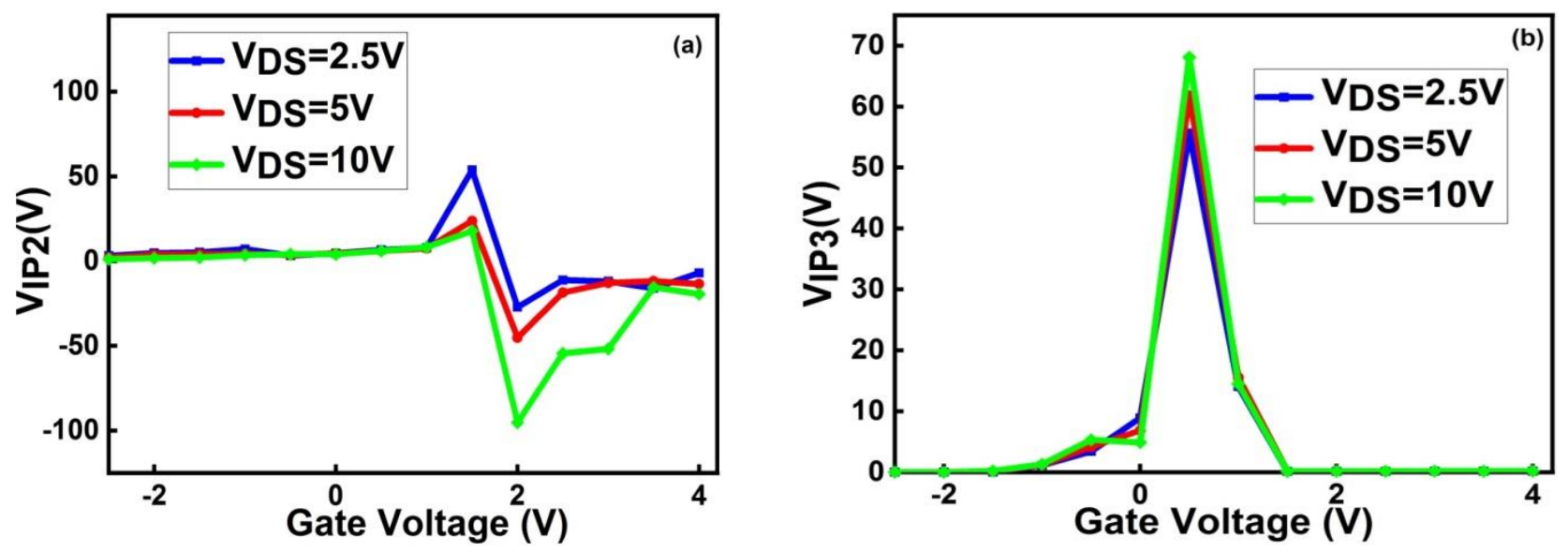

Fig.7 High linearity parameters analysis of gate recessed MOSHEMT for different drain voltages (a) VIP2- $V_{g}$ characteristic curves (b) $V_{\text {IP3 }}-V_{g}$ characteristic curves

Input Intercept point of the third-harmonic (IIP3) and Intermodulation distortion of thirdharmonic (IDM3) with respect to gate-to-source voltage is demonstrated by Fig.8

Carrier transport efficiency and better gate control over the channel leads to improve the IIP3. When drain-to-source voltage increases IIP3 also shows better improvement.
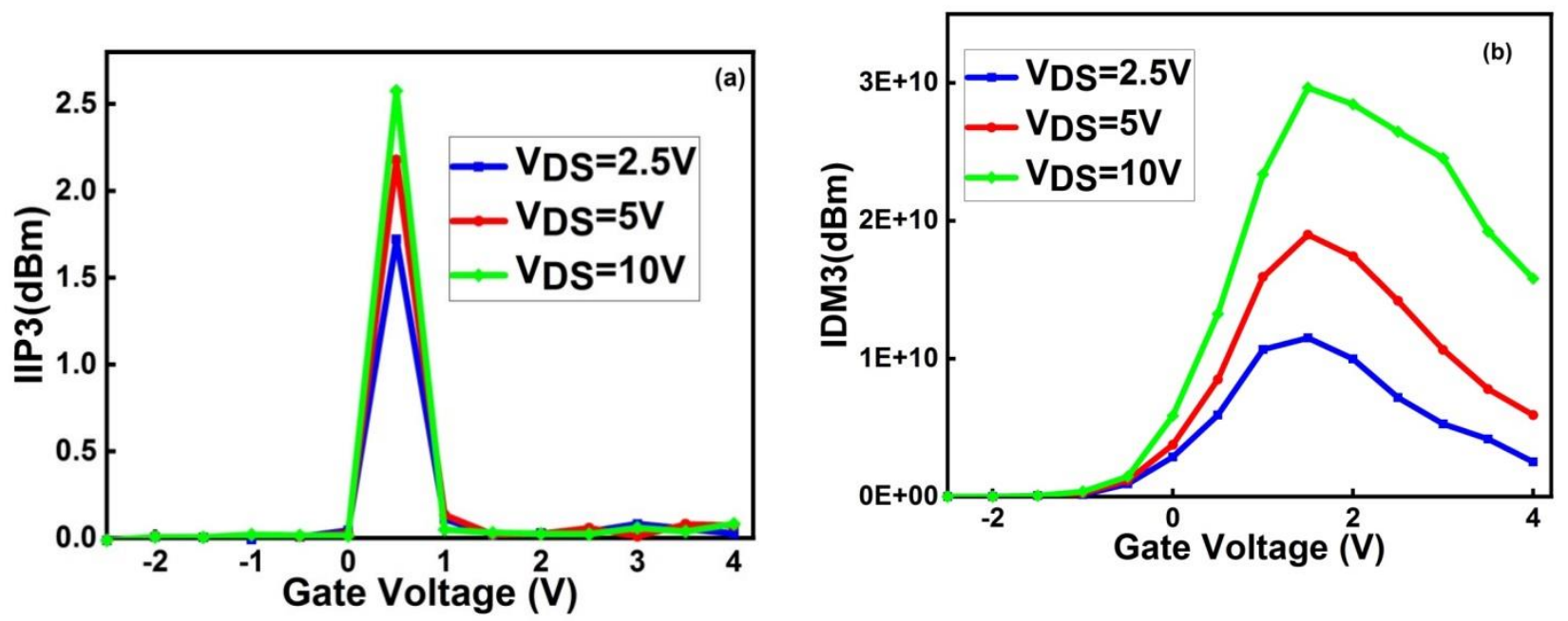

Fig.8 High linearity parameters analysis of gate recessed MOSHEMT for different drain voltages (a) IIP3- $V_{g}$ characteristic curves (b) IDM3-V $\mathrm{V}_{\mathrm{g}}$ characteristic curves

The comparative analysis of gate recessed MOSHMET for different drain-to-source voltages is shown in Table1. It is clearly understood from the table that gate recessed MOSHEMT shows better results for $\mathrm{V}_{\mathrm{ds}}=10 \mathrm{~V}$ due sustainable increment in higher current density and transconductance. 
Table 1: AIGaN/GaN gate recessed MOSHEMT simulation Results for different drain-tosource voltages

\begin{tabular}{|l|l|l|c|c|c|}
\hline S.No. & \multicolumn{1}{|c|}{ Parameters } & Unit & $\mathbf{V}_{\mathbf{d s}}=\mathbf{2 . 5 V}$ & $\mathbf{V}_{\mathbf{d s}}=\mathbf{5 V}$ & $\mathbf{V}_{\mathrm{ds}}=\mathbf{1 0 V}$ \\
\hline 1. & Threshold voltage & $\mathrm{V}$ & 0.5 & 0.5 & 0.5 \\
\hline 2. & Current density & $\mathrm{mA} / \mathrm{mm}$ & 481 & 673 & 888 \\
\hline 3. & Trans conductance & $\mathrm{mS} / \mathrm{mm}$ & 140 & 180 & 225 \\
\hline 4. & Gate-to-source capacitance & $\mathrm{pF}$ & 38.75 & 40.65 & 42.75 \\
\hline 5. & Cut-off Frequency & $\mathrm{GHz}$ & 0.50 & 0.69 & 0.91 \\
\hline 6. & Max. Oscillation Freq. & $\mathrm{Hz}$ & $6.81 \times 10^{-6}$ & $7.45 \times 10-6$ & $8.52 \times 10-6$ \\
\hline 7. & VIP2 & $\mathrm{V}$ & 53.77 & 23.70 & 17.87 \\
\hline $\mathbf{8 .}$ & VIP3 & $\mathrm{V}$ & 55.64 & 61.80 & 68.07 \\
\hline 9. & IIP3 & $\mathrm{dBm}$ & 1.72 & 2.17 & 2.57 \\
\hline $\mathbf{1 0}$. & IDM3 & $\mathrm{dBm}$ & $1.50 \times 10^{10}$ & $1.89 \times 10^{10}$ & $2.96 \times 10^{10}$ \\
\hline
\end{tabular}

\section{CONCLUSION}

In this work, a comparative analysis of analog/RF parameters for AlGaN/GaN HEMT, MOSHEMT and Gate recessed MOSHEMT is presented which may provide a basic idea for newly researcher for starting research in this field. Analog/RF and High linearity parameters of AlGaN/GaN on-state (HEMT), off-state (MOSHEMT) and Gate recessed MOSHEMT have been investigated by developing analytical models and the results has been verified using simulations. The both on-state and Off-State devices are very much suited for GaN RF and power device applications. The addition of $\mathrm{Al}_{2} \mathrm{O}_{3}$ oxide layer between the schottky-gate and AlGaN barrier-layer in the Off-state device structure improve the on current and also reduce the gate leakage current as compare to the on-state device structure. For high power applications modified gate recessed MOSHEMT structure is best suited on HEMT and MOSHMET. In comparison HEMT and MOSHEMT devices, gate recessed MOSHEMT shows better improvement in terms of gate leakage current, transconductance, breakdown voltage, 2-DEG charge density, gate to drain capacitance, $\mathrm{I}_{\mathrm{on}} / \mathrm{I}_{\text {off }}$ ratio and also it shows a better improvement in terms of high linearity parameters of $\mathrm{V}_{\text {IP2 }}, \mathrm{V}_{\text {IP3 }}$, IIP3 and IDM3.

Acknowledgements: Not applicable.

Author Contributions: The authors have contributed mutually regarding this paper.

Funding: Not applicable.

Data Availability: All data available within the manuscript.

\section{Declaration}

Ethics Approval and Consent to Participate The authors declared that the manuscript ethics is approved as per the journal. 
Consent for Publication: The authors give full consent for the publication of this research work.

Competing interests: Not applicable.

Conflict of interest: The authors declare that they have no conflict of interest.

Research Involving Human Participants and/or Animals: Not applicable.

Informed Consent: Not applicable.

\section{REFERENCES}

1. Kim, Hyun-Seop, Myoung-Jin Kang, Jeong Jin Kim, Kwang-Seok Seo, and Ho-Young Cha. "Effects of Recessed-Gate Structure on AlGaN/GaN-on-SiC MIS-HEMTs with Thin AlOxNy MIS Gate." Materials 13, no. 7 (2020): 1538.

2. Basu, Sarbani, Pramod K. Singh, Po-Wen Sze, and Yeong-Her Wang. "AlGaN/GaN metal-oxidesemiconductor high electron mobility transistor with liquid phase deposited $\mathrm{Al} 2 \mathrm{O} 3$ as gate dielectric." Journal of The Electrochemical Society 157, no. 10 (2010): H947.

3. Hasan, Md Rezaul, Abhishek Motayed, Md Shamiul Fahad, and Mulpuri V. Rao. "Fabrication and comparative study of DC and low frequency noise characterization of GaN/AlGaN based MOS-HEMT and HEMT." Journal of Vacuum Science \& Technology B, Nanotechnology and Microelectronics: Materials, Processing, Measurement, and Phenomena 35, no. 5 (2017): 052202.

4. Lee, Ching-Ting, Jhe-Hao Chang, and Chun-Yen Tseng. "Monolithic enhancement-mode and depletion-mode GaN-based MOSHEMTs." In Gallium Nitride Materials and Devices XI, vol. 9748, p. 97480Z. International Society for Optics and Photonics, 2016.

5. Taube, Andrzej, Mariusz Sochacki, Jan Szmidt, Eliana Kamińska, and Anna Piotrowska. "Modelling and Simulation of Normally-Off AlGaN/GaN MOS-HEMTs." International Journal of Electronics and Telecommunications 60, no. 3 (2014): 253-258.

6. Huang, Kuan Ning, Yueh Chin Lin, Jin Hwa Lee, Chia Chieh Hsu, Jing Neng Yao, Chieh Ying Wu, Chao Hsin Chien, and Edward Yi Chang. "Study of tri-gate AlGaN/GaN MOS-HEMTs for power application." Micro and Nano Engineering (2020): 100073.

7. Verma, Sumit, Sajad A. Loan, Abdulrahman M. Alamoud, and Abdullah G. Alharbi. "Hybrid AlGaN/GaN high-electron mobility transistor: design and simulation." IET Circuits, Devices \& Systems 12, no. 1 (2018): 33-39.

8. Yuan-Zheng, Yue, Hao Yue, Zhang Jin-Cheng, Feng Qian, Ni Jin-Yu, and Ma Xiao-Hua. "A study on $\mathrm{Al}_{2} \mathrm{O}_{3}$ passivation in GaN MOS-HEMT by pulsed stress." Chinese Physics B 17, no. 4 (2008): 1405.

9. Meng, Di, Shuxun Lin, Cheng P. Wen, Maojun Wang, Jinyan Wang, Yilong Hao, Yaohui Zhang, Kei May Lau, and Wengang Wu. "Low leakage current and high-cutoff frequency AlGaN/GaN MOSHEMT using submicrometer-footprint thermal oxidized $\mathrm{TiO}_{2} / \mathrm{NiO}$ as gate dielectric." IEEE electron device letters 34, no. 6 (2013): 738-740.

10. Touati, Zine-eddine, Zahra Hamaizia, and Zitouni Messai. "DC and RF characteristics of AlGaN/GaN HEMT and MOS-HEMT." In 2015 4th International Conference on Electrical Engineering (ICEE), pp. 1-4. IEEE, 2015.

11. Chakroun, Ahmed, Abdelatif Jaouad, Meriem Bouchilaoun, Osvaldo Arenas, Ali Soltani, and Hassan Maher. "Normally-off AlGaN/GaN MOS-HEMT using ultra-thin Al0. 45Ga0. 55N barrier layer." physica status solidi (a) 214, no. 8 (2017): 1600836.

12. Sandeep, V., J. Charles Pravin, A. Ramesh Babu, and P. Prajoon. "Impact of AlInN Back-Barrier Over AlGaN/GaN MOS-HEMT With $\mathrm{HfO}_{2}$ Dielectric Using Cubic Spline Interpolation Technique." IEEE Transactions on Electron Devices 67, no. 9 (2020): 3558-3563.

13. Wu, Tian-Li, Shun-Wei Tang, and Hong-Jia Jiang. "Investigation of recessed gate AlGaN/GaN MIS-HEMTs with double AlGaN barrier designs toward an enhancement-mode characteristic." Micromachines 11, no. 2 (2020): 163.

14. Yue, Yuanzheng, Yue Hao, Jincheng Zhang, Jinyu Ni, Wei Mao, Qian Feng, and Linjie Liu. "AlGaN/GaN MOS-HEMT With $\mathrm{HfO}_{2}$ Dielectric and $\mathrm{Al}_{2} \mathrm{O}_{3}$ Interfacial Passivation Layer Grown by Atomic Layer Deposition." IEEE electron device letters 29, no. 8 (2008): 838-840. 
15. Jena, Kanjalochan, Raghunandan Swain, and Trupti Ranjan Lenka. "Modeling and comparative analysis of DC characteristics of $\mathrm{AlGaN} / \mathrm{GaN}$ HEMT and MOSHEMT devices." International Journal of Numerical Modelling: Electronic Networks, Devices and Fields 29, no. 1 (2016): 83-92.

16. Roccaforte, Fabrizio, Giuseppe Greco, Patrick Fiorenza, and Ferdinando Iucolano. "An overview of normally-off GaN-based high electron mobility transistors." Materials 12, no. 10 (2019): 1599.

17. Saito, Wataru, Yoshiharu Takada, Masahiko Kuraguchi, Kunio Tsuda, and Ichiro Omura. "Recessed-gate structure approach toward normally off high-voltage AlGaN/GaN HEMT for power electronics applications." IEEE Transactions on electron devices 53, no. 2 (2006): 356-362.

18. Tapajna, Milan, and Ján Kuzmík. "Control of threshold voltage in $\mathrm{GaN}$ based metal-oxide-semiconductor high-electron mobility transistors towards the normally-off operation." Japanese Journal of Applied Physics 52, no. 8S (2013): 08JN08.

19. Zhou, Hong, Xiabing Lou, Karynn Sutherlin, Jarren Summers, Sang Bok Kim, Kelson D. Chabak, Roy G. Gordon, and D. Ye Peide. "DC and RF performance of AlGaN/GaN/SiC MOSHEMTs with deep sub-micron T-gates and atomic layer epitaxy $\mathrm{MgCaO}$ as gate dielectric." IEEE Electron Device Letters 38, no. 10 (2017): $1409-1412$.

20. Husna, Fatima, Mohamed Lachab, Mahbuba Sultana, Vinod Adivarahan, Qhalid Fareed, and Asif Khan. "High-Temperature Performance of AlGaN/GaN MOSHEMT With $\mathrm{SiO}_{2}$ Gate Insulator Fabricated on $\mathrm{Si}$ (111) Substrate." IEEE Transactions on Electron Devices 59, no. 9 (2012): 2424-2429.

21. Mazumder, Soumen, Ssu-Hsien Li, Zhan-Gao Wu, and Yeong-Her Wang. "Combined implications of UV/O3 interface modulation with HfSiOX surface passivation on AlGaN/AIN/GaN MOS-HEMT." Crystals 11, no. 2 (2021): 136.

22. Wu, Jianzhi, Wei Lu, and K. L. Paul. "Normally-OFF AlGaN/GaN MOS-HEMT with a two-step gate recess." In 2015 IEEE International Conference on Electron Devices and Solid-State Circuits (EDSSC), pp. 594-596. IEEE, 2015.

23. Swain, R., K. Jena, A. Gaini, and T. R. Lenka. "Comparative study of AlN/GaN HEMT and MOSHEMT structures by varying oxide thickness." In 2014 IEEE 9th Nanotechnology Materials and Devices Conference (NMDC), pp. 128-131. IEEE, 2014.

24. Verma, Manish, and Ashutosh Nandi. "Design and Analysis of AlGaN/GaN Based DG MOSHEMT for HighFrequency Application." Transactions on Electrical and Electronic Materials 21, no. 4 (2020): 427-435.

25. Hao, Yue, Ling Yang, Xiaohua Ma, Jigang Ma, Menyi Cao, Caiyuan Pan, Chong Wang, and Jincheng Zhang. "High-performance microwave gate-recessed AlGaN/AlN/GaN MOS-HEMT with 73\% power-added efficiency." IEEE Electron Device Letters 32, no. 5 (2011): 626-628.

26. Chakrabarty, A., and R. Swain. "Modelling of fin width dependent threshold voltage in fin shaped nano channel AlGaN/GaN HEMT." Superlattices and Microstructures 141 (2020): 106497.

27. Zine-eddine, Touati, Hamaizia Zahra, and Messai Zitouni. "Design and analysis of $10 \mathrm{~nm}$ T-gate enhancement-mode MOS-HEMT for high power microwave applications." Journal of Science: Advanced Materials and Devices 4, no. 1 (2019): 180-187.

28. Silvaco: ATLAS user's manual: device simulation Software (Silvaco, Santa Clara, CA, USA, 2013)

29. Vurgaftman, I., J. áR Meyer, and L. áR Ram-Mohan. "Band parameters for III-V compound semiconductors and their alloys." Journal of applied physics 89, no. 11 (2001): 5815-5875.

30. Moses, Poul Georg, Maosheng Miao, Qimin Yan, and Chris G. Van de Walle. "Hybrid functional investigations of band gaps and band alignments for AlN, GaN, InN, and InGaN." The Journal of chemical physics 134, no. 8 (2011): 084703.

31. Angerer, H., D. Brunner, F. Freudenberg, O. Ambacher, M. Stutzmann, R. Höpler, T. Metzger et al. "Determination of the $\mathrm{Al}$ mole fraction and the band gap bowing of epitaxial $\mathrm{Al}{ }_{\mathrm{x}} \mathrm{Ga}_{1-} \mathrm{N}$ films." Applied Physics Letters 71, no. 11 (1997): 1504-1506.

32. Piprek, Joachim. Semiconductor optoelectronic devices: introduction to physics and simulation. Elsevier, 2013.

33. Ambacher, Oliver, B. Foutz, Joseph Smart, J. R. Shealy, N. G. Weimann, K. Chu, M. Murphy et al. "Two dimensional electron gases induced by spontaneous and piezoelectric polarization in undoped and doped AlGaN/GaN heterostructures." Journal of applied physics 87, no. 1 (2000): 334-344.

34. Yigletu, Fetene Mulugeta, Sourabh Khandelwal, Tor A. Fjeldly, and Benjamin Iniguez. "Compact chargebased physical models for current and capacitances in AlGaN/GaN HEMTs." IEEE Transactions on Electron Devices 60, no. 11 (2013): 3746-3752.

35. Farahmand, Maziar, Carlo Garetto, Enrico Bellotti, Kevin F. Brennan, Michele Goano, Enrico Ghillino, Giovani Ghione, John D. Albrecht, and P. Paul Ruden. "Monte Carlo simulation of electron transport in the III-nitride wurtzite phase materials system: binaries and ternaries." IEEE Transactions on electron devices 48, no. 3 (2001): 535-542. 
36. Braga, N., R. Mickevicius, R. Gaska, X. Hu, M. S. Shur, M. Asif Khan, Grigory Simin, and J. Yang. "Simulation of hot electron and quantum effects in $\mathrm{AlGaN} / \mathrm{GaN}$ heterostructure field effect transistors." Journal of applied physics 95, no. 11 (2004): 6409-6413.

37. Jena, Kanjalochan, Raghunandan Swain, and Trupti Ranjan Lenka. "Effect of thin gate dielectrics on DC, radio frequency and linearity characteristics of lattice-matched AlInN/AlN/GaN metal-oxide-semiconductor high electron mobility transistor." IET Circuits, Devices \& Systems 10, no. 5 (2016): 423-432.

38. Ghosh, Sneha, Anindita Mondal, Mousiki Kar, and Atanu Kundu. "Study of Effective Graded Oxide Capacitance and Length Variation on Analog, RF and Power Performances of Dual Gate Underlap MOSHEMT." (2021).

39. Freedsman, Joseph Jesudass, Toshiharu Kubo, and Takashi Egawa. "High Drain Current Density E-Mode Al2O3/AlGaN/GaN MOS-HEMT on Si with Enhanced Power Device Figure-of-Merit $\left(4 \mathrm{X}_{10} 0^{8} \mathrm{~V}^{2} \Omega^{-1} \mathrm{~cm}^{-}\right.$ 2)." IEEE transactions on electron devices 60, no. 10 (2013): 3079-3083. 